\title{
Enhancing the Quality of Underwater Images using Fusion of sequential Filters and Dehazing
}

\author{
M.Suganthy", S. Lakshmi, S.Palanivel \\ Vel Tech Multitech Dr.Rangarajan Dr. Sakunthala Engineering College, Chennai, Tamil Nadu, India \\ *Corresponding Author E-mail: Suganthym46@gmail.com
}

\begin{abstract}
Effectively analyzing underwater images and identifying any object under the water has become a difficult task. Generally, the factors affecting underwater images are uneven lighting, low contrast, blunt colors, and characteristics of an object based on absorption and scattering of light. The proposed technique involves applying white balancing and contrast enhancement to the original image. The combination of filters namely homomorphic filtering, wavelet denoising, bilateral filter, adaptive filters are used and applied sequentially on the degraded underwater images. The results obtained showed that the proposed algorithm works well in refining the underwater image attributes. Peak Signal to Noise Ratio (PSNR) and Mean Squared Error (MSE) are used to evaluate performance of the algorithm.
\end{abstract}

Keywords: PSNR, MSE, White Balance, Contrast stretching, and Adaptive Median Filter.

\section{Introduction}

Now a days the analyzing underwater images has become an interesting field. Focusing on the enhancement of underwater images has vital role in executing the significance of nature and environment.[1-3]. In general the methods restoration and enhancement used to lower the degradation. [ 4-5]. Both restoration and enhancement have significance effect in improving the image performance individually. Several techniques were developed to upgrade the attributes of an underwater image [611]. The design of unique hardware and the use of multiple images of same scene can be exploited to reduce the hurdles of underwater image enhancement.

In process of the image appearance enhancement white balancing technique plays vital role that discards unwanted color effects. The contrast of the image is enhanced using histogram stretching which results in providing clear haze region also yields some degradation. These degraded parts can be removed by applying weight maps. Three weight maps are used that are: Luminance, Chromaticity and saliency. To compensate the loss of color and contrast in result of luminance, the Chromaticity and saliency are followed. In the Chromatic Weight Map, the amount of saturation of the image is obtained while the Saliency Weight Map provides relationship between a region with respect to its neighborhood regions. Though various techniques are presented to enhance the characteristics of underwater images, still there exists some problems. Research focus on addressing the number of issues that reduce their practical applicability.

In this work, by applying white balancing and the sequential filters the unwanted attributes exist in the image are reduced comparable. The information obtained from the image are fused to reinforce features. The input obtained through white balancing technique and contrast enhancement filters are fused to provide better image. The outputs obtained are free from haze and noise with improved PSNR.

\section{Proposed System}

In this proposed system in Fig 1, dehazing and denoising process are performed using the sequential process of homomorphic filtering, wavelet denoising, bilateral filter and adaptive median filter[13].

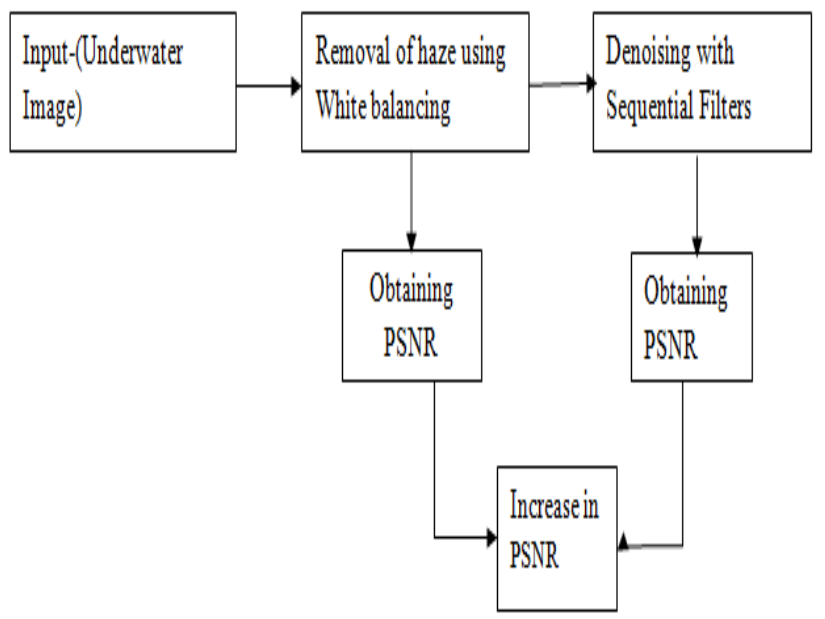

Fig. 1: System Flow Diagram

The noise present in the dehazed image is reduced considerably. Median filter is applied to preserve edges and to produce the output with enhanced visibility and color.

\section{Image Dehazing}

White balancing is used to make image better by detaching excluded colors obtained in the result of attenuation. The white balancing stage aims at removing the color cast induced by underwater light scattering, so as to process the sea images while 
preserving its natural characteristics . Since white balancing technique is not sufficient to reduce hazing effects, it follows sequential filtering process[12 \&15].

\section{Image Denoising}

The process of eliminating noise exist in the image is known as Image denoising. At first Image denoising is performed with the use of homomorphic filter to compensate the effects of uneven lighting meanwhile the image features are also enhanced. Then removal of noise is by wavelet transform[14] which includes modification of wavelet coefficients based on predefined criteria and inverse transform is performed on these modified coefficients. Finally Adaptive median filter is used here which

replaces the corrupted pixel by median value. This filter works well in removing impulse noise preserving the edges.

In general the image is affected by various noises during transmission and these are measured by the use of Peak Signal to Noise Ratio (PSNR) PSNR

The PSNR (in $\mathrm{dB}$ ) is calculated as:

$$
P S N R=10 \log _{10} \frac{255^{2}}{\mathrm{MSE}}
$$

\section{Results and Discussion}

The results obtained for proposed algorithms are depicted in the figures 2 and 3.
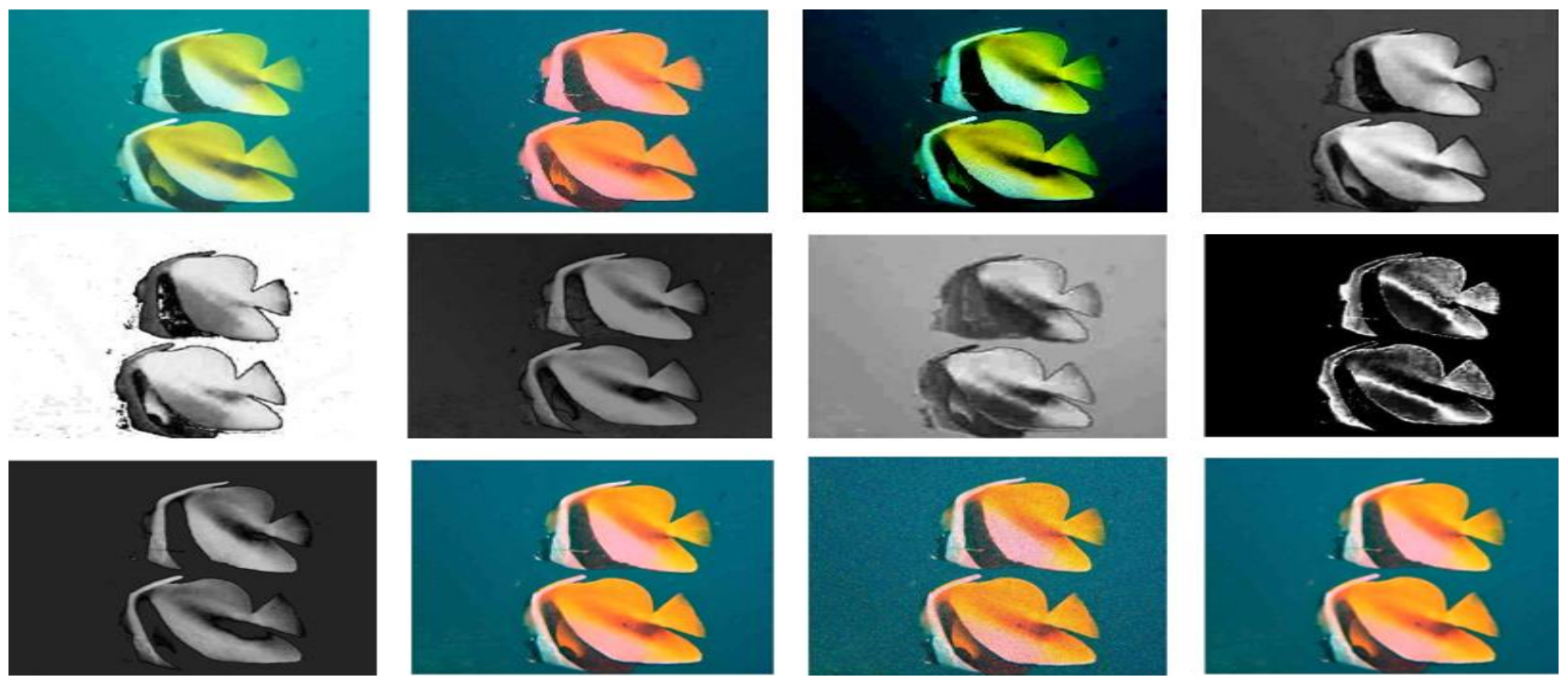

Figure 2: Image with free of noise and haze
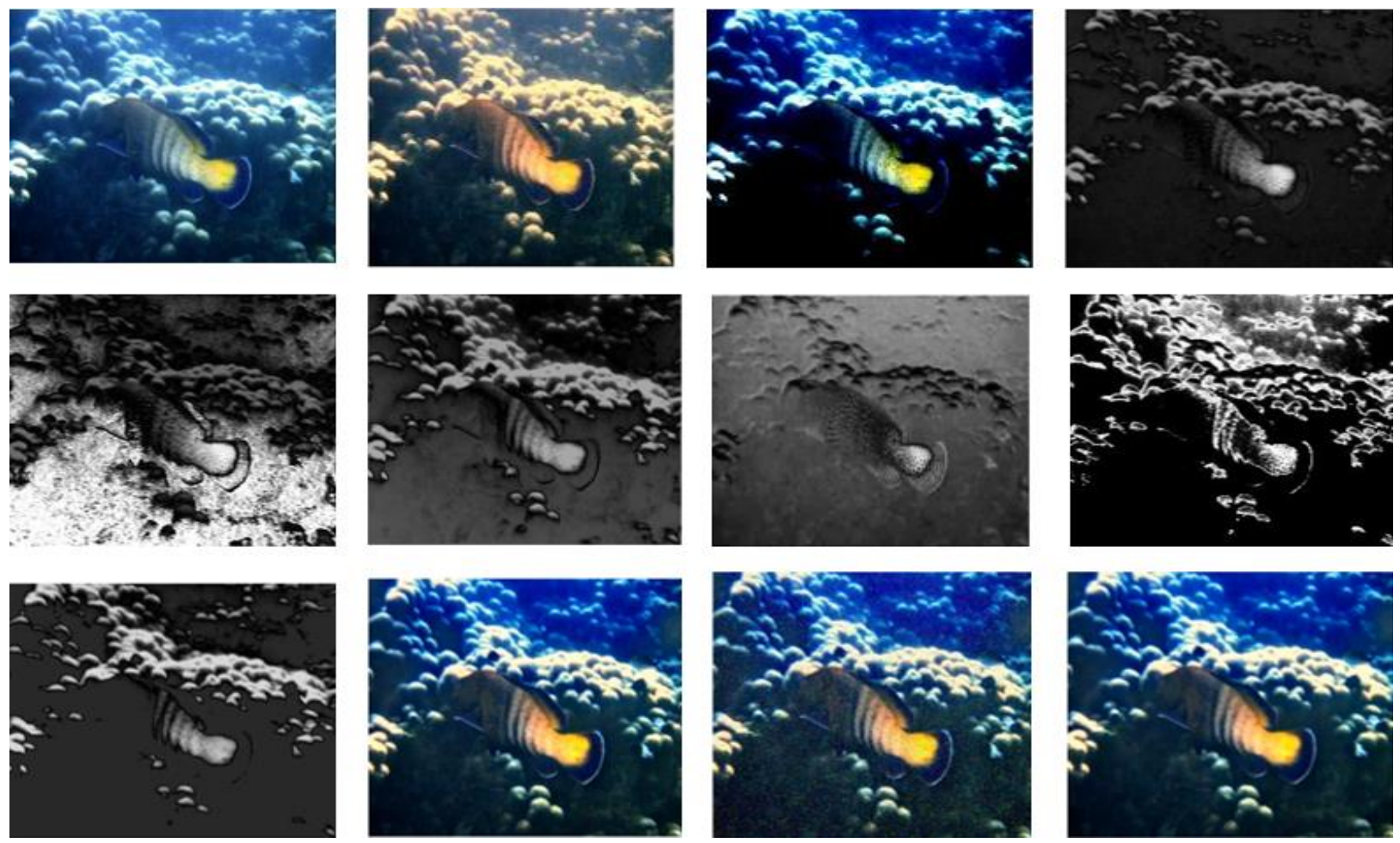

Fig. 3: Image with free of noise and haze

The PSNR values of dehazed noisy image and dehazed noiseless image is is given in Table I. It shows that the proposed sysmem works well in improving PSNR value. 
Table 1: PSNR values

\begin{tabular}{|c|c|c|c|c|c|}
\hline S.No & Fig.No & Gray value & $\begin{array}{c}\text { PSNR value for haze } \\
\text { free noisy image }(\mathrm{db})\end{array}$ & $\begin{array}{c}\text { PSNR value for noise free } \\
\text { hazy free image }(\mathrm{db})\end{array}$ & $\begin{array}{c}\text { Percentage increase in } \\
\text { PSNR in proposed system. } \\
(\% \mathrm{db})\end{array}$ \\
\hline 1 & 3 & 0.3521 & 69.2156 & 77.3059 & 11.2437 \\
\hline 2 & 4 & 0.4717 & 69.1721 & 87.0327 & 24.9291 \\
\hline
\end{tabular}

\section{Conclusion and Future Work}

The simulation result clearly shows that the algorithm used to extract image with free of haze and noise outperforms by preserving the contents present in the image. PSNR is calculated for the fused image (dehazed and denoised) which gives noticeable increase in the PSNR value. Though the proposed method achieved better result, it will not be good in providing images with free of noise and haze. Future work is to address the effects of noise more effectively and to give clear way for analyzing the image for specific application.

\section{References}

[1] Schechner Y Y, Karpel N, 2004, ' Clear underwater vision[C]//Computer Vision and Pattern Recognition', CVPR 2004. Proceedings of the IEEE Computer Society Conference on IEEE, 2, vol. 1,no.1, pp. I-536-I-543.

[2] Kocak D M, Caimi F M. The current art of underwater imagingWith a glimpse of the past and vision of the future[J]. Marine Technology Society Journal, 2005, vol.39, no.3, pp. 5-26.

[3] Raimondo S, Silvia C. 2010,'Underwater image processing: state of the art of restoration and image enhancement methods' [J]. EURASIP Journal on Advances in Signal Processing,

[4] Prabhakar C J and Praveen Kumar P U (2010), in Proceedings of International Conference on Signal and Image Processing, pp.322327.

[5] Prabhakar C.J.1*, Praveen Kumar P.U. 'An Image Based Technique For Enhancement Of Underwater Images 'International Journal of Machine Intelligence ISSN: 0975-2927 \& E-ISSN: 0975-9166, Volume 3, Issue 4, 2011, pp-217-224.

[6] Iqbal K, Abdul Salam R, Osman M, et al., 2007, ' Underwater Image Enhancement Using An Integrated Colour Model[J]', IAENG International Journal of Computer Science, , vol.32, no.2 pp.239-244.

[7] Petit F, Capelle-Laize A, Carré P. 2009,' Underwater image enhancement by attenuation inversionwith quaternions[C]', Acoustics, Speech and Signal Processing, 2009. ICASSP 2009. IEEE International Conference on. IEEE,: 1177-1180.

[8] Schechner Y Y, Karpel N, 2005, ' Recovery of underwater visibility and structure by polarization analysis[J]', Oceanic Engineering, IEEE Journal, vol.30, no.3, pp.570-587.

[9] Narasimhan S G, Nayar S K, 2003, "Contrast restoration of weather degraded images[J]', Pattern Analysis and Machine Intelligence, IEEE Transactions on, vol.25, no.6, pp. 713-724

[10] He D M, Seet G G L, 2004, 'Divergent-beam lidar imaging in turbid water[J]', . Optics and lasers in engineering, vol.41, no.1, pp.217-231.

[11] Schechner Y Y, Averbuch Y, 2007, ' Regularized image recovery in scattering media[J]'. Pattern Analysis and Machine Intelligence, IEEE Transactions on, vol. 29,no.9, pp. 1655-1660.

[12] CodrutaOrnianaAncuti and Cosmin Ancuti, (2013)“Single Image Dehazing By Multi-Scale Fusion" IEEE Transactions On Image Processing, vol. 22, no. 8,

[13] M.Suganthy,P.Ramamoorthy, 2012, 'An Efficient authentication By Iris Using Log Gabor Filter and Neural network' , IJCS International Journal of Computer Science Issues, vol. 9, no.5, pp.1694-0814

[14] M. Suganthy ,P. Ramamoorthy and A. Kanagalakshmi, 2014 'Wavelet And Texture Feature Fusion Of Dual IrisBased Security System Using T-Norm' vol. 9, no. 24 ,pp. 30681-30692

[15] Codruta O. Ancuti , Cosmin Ancuti, Christophe De Vleeschouwer and Philippe Bekaert, JANUARY 2018 'Color Balance and Fusion for Underwater Image Enhancement' IEEE Transactions On Image Processing, VOL. 27, NO. 1, pp-379-393

[16] T.Padmapriya and V.Saminadan, "Utility based Vertical Handoff Decision Model for LTE-A networks", International Journal of
Computer Science and Information Security, ISSN 1947-5500, vol.14, no.11, November 2016

[17] S.V.Manikanthan and K.srividhya "An Android based secure access control using ARM and cloud computing", Published in: Electronics and Communication Systems (ICECS), 2015 2nd International Conference on 26-27 Feb. 2015 Publisher: IEEE,DOI: 10.1109/ECS.2015.7124833. 